\title{
The Effects of Dietary Glycine on the Acetic Acid-Induced Mouse Model of Colitis
}

\author{
Xin Wu $\mathbb{D}^{1},{ }^{1}$ Yongmin Zheng $\left(\mathbb{D},{ }^{1}\right.$ Jie Ma $\mathbb{D}^{1},{ }^{1}$ Jie Yin $\mathbb{D}^{1},{ }^{1}$ and Shuai Chen $\mathbb{D}^{1,2}$ \\ ${ }^{1}$ College of Animal Science and Technology, Hunan Agriculture University, Changsha, China; Hunan Co-Innovation Center of \\ Animal Production Safety, Changsha, Hunan 410128, China \\ ${ }^{2}$ Scientific Observing and Experimental Station of Animal Nutrition and Feed Science in South-Central, Ministry of Agriculture, \\ Hunan Provincial Engineering Research Center of Healthy Livestock, Key Laboratory of Agro-Ecological Processes in \\ Subtropical Region, Institute of Subtropical Agriculture, Chinese Academy of Sciences, Changsha, Hunan 410125, China
}

Correspondence should be addressed to Jie Yin; yinjie2014@126.com and Shuai Chen; chenshuai@mail.com

Received 14 May 2020; Revised 3 July 2020; Accepted 9 July 2020; Published 5 August 2020

Guest Editor: Xiaolu Jin

Copyright (C) 2020 Xin Wu et al. This is an open access article distributed under the Creative Commons Attribution License, which permits unrestricted use, distribution, and reproduction in any medium, provided the original work is properly cited.

\begin{abstract}
Inflammatory bowel disease, a gut disease that is prevalent worldwide, is characterized by chronic intestinal inflammation, such as colitis, and disorder of the gut microbiome. Glycine (Gly) is the simplest amino acid and functions as an anti-inflammatory immune-nutrient and intestinal microbiota regulator. This study aimed at investigating the effect of Gly on colitis induced in mice by intrarectal administration of $5 \%$ acetic acid (AA). Bodyweight and survival rates were monitored, and colonic length and weight, serum amino acid concentrations, intestinal inflammation-related gene expression, and colonic microbiota abundances were analyzed. The results showed that Gly dietary supplementation had no effect on the survival rate or the ratio of colonic length to weight. However, Gly supplementation reversed the AA-induced increase in serum concentrations of amino acids such as glutamate, leucine, isoleucine, and valine. Furthermore, Gly inhibited colonic gene expression of interleukin- (IL-) $1 \beta$ and promoted IL-10 expression in colitis mice. Gly supplementation also reversed the AA-induced reduction in the abundance of bacteria such as Clostridia, Ruminococcaceae, and Clostridiales. This change in the intestinal microbiota was possibly attributable to the changes in colonic IL-10 expression and serum concentrations of valine and leucine. In sum, Gly supplementation regulated the serum concentrations of amino acids, the levels of colonic immune-associated gene expression, and the intestinal microbiota in a mouse model of colitis. These findings enhance our understanding of the role of Gly in regulating metabolism, intestinal immunity, and the gut microbiota in animals afflicted with colitis.
\end{abstract}

\section{Introduction}

Inflammatory bowel disease (IBD), including Crohn's disease (CD) and ulcerative colitis (UC), is characterized by a loss of intestinal mucosal homeostasis associated with inappropriate and aggravated immune responses to intestinal lumen antigens [1-3]. The clinical symptoms of IBD include diarrhea, abdominal pain, bloody stools, tenderness, and abdominal mass. The increased incidence of IBD is a worldwide healthcare problem [4]. It is widely believed that IBD is caused by complex interactions between genetic and environmental factors, such as immune-response disorders and microbial community changes. However, the cause of IBD remains unclear, and further research is needed $[5,6]$.
Several types of pharmacologically induced animal colitis models have been developed, such as dextran sulfate- (DSS-), trinitrobenzene sulfonic acid- (TNBS-), or acetic acid- (AA-) induced models $[7,8]$. DSS is widely used for this purpose, as is TNBS, typically in combination with ethanol. AA causes UC (hereafter termed "colitis") by damaging the intestinal mucosa epithelium and is a low-cost and convenient technique. Previous studies demonstrated that AA-induced colitis might be a good model with which to study the efficacy of drugs [9], so we used AA to induce colitis in this study.

Anti-inflammatory drugs such as cytokine antagonists and immunosuppressive drugs such as corticosteroids are currently used for IBD treatment [10-12]. However, longterm use of cytokine antagonists may have severe side 
Table 1: Primers used in this study.

\begin{tabular}{llrc}
\hline Gene & ID & Nucleotide sequence of primers $\left(5^{\prime}-3^{\prime}\right)$ & Product length \\
\hline IL-1 $\beta$ & NM_008361.3 & ATGAAAGACGGCACACCCAC GCTTGTGCTCTGCTTGTGAG & 175 \\
IL-17 & NM_010552.3 & TACCTCAACCGTTCCACGTC TTTCCCTCCGCATTGACAC & 119 \\
IL-10 & NM_010548.2 & GCCACATGCTCCTAGAGCTG CAGCTGGTCCTTTGTTTGAAA & 71 \\
IFN- $\gamma$ & NM_008337.4 & ATGAACGCTACACACTGCATCTTGGCTT & 361 \\
Actin & NM_007393.5 & CCTCAAACTTGGCAATACTCATGATGC & 366 \\
\hline
\end{tabular}

effects [13]. Therefore, new adjuvant therapies are required to overcome the limitations of current drug therapies [14].

Many amino acids have been shown to have beneficial effects in IBD. For example, arginine reduces mucosal permeability, inhibits inflammation, and increases inducible nitric oxide synthase (iNOS) activity in mice with DSS-induced colitis [15]. It has also been shown that Gly may inhibit cytokine secretion in monocytes, macrophages, and neutrophils, and regulate $\mathrm{T}$ cell activities [16]. Dietary Gly prevents diarrhea, bodyweight loss, and ulceration in rats with DSS-induced IBD $[17,18]$. However, the effects of Gly on AA-induced IBD in mice remain unclear.

This study aimed at investigating the effect of Gly on AAinduced colitis in mice. The results add to our knowledge of a possible role for Gly in colitis treatment.

\section{Materials and Methods}

2.1. Animal Model. This study was conducted following the guidelines of the Animal Welfare Committee of the Institute of Subtropical Agriculture, Chinese Academy of Sciences (2015-8A). Female six-week-old ICR (Institute for Cancer Research) mice were purchased from the SLAC laboratory animal center (Changsha, China). The mice were housed in a temperature-controlled environment with a $12 \mathrm{~h}$ light/dark cycle and had free access to food and water. Sixty mice were randomly divided into three groups of 20 animals: a control (Con) group (fed the basal diet), a model (Mod) group (fed the basal diet), and a model+Gly (ModGly) group (fed the basal diet supplemented with $0.1 \%$ Gly). The dose of Gly was selected following preliminary experiments. The Con group and Mod group shared the data with our previous study [19]. On the seventh day of the experiment, all of the mice were fasted for $36 \mathrm{~h}$ and were intrarectally treated with 5\% AA (Mod and ModGly group) or saline solution (Con group). The surviving mice were monitored for 1 week after the administration of AA, and all of the mice were then sacrificed by cervical dislocation, and blood, colons, and colonic contents were collected.

2.2. Analysis of Serum Amino Acids. High-performance liquid chromatography (HPLC) was used to analyze serum amino acids. Briefly, $0.1 \mathrm{ml}$ of the serum sample was thoroughly mixed with $4.9 \mathrm{ml}$ of $0.01-\mathrm{M}$ hydrochloric acid and then centrifuged at $5000 \mathrm{rpm}$ and $4^{\circ} \mathrm{C}$ for $5 \mathrm{~min}$. The supernatant $(500 \mathrm{ml})$ was then removed and incubated at $4^{\circ} \mathrm{C}$ for $12 \mathrm{~h}$ and then homogenized with $8 \%$ salicylic acid. The resulting homogenate was centrifuged twice at $12,000 \mathrm{rpm}$ and $4^{\circ} \mathrm{C}$ for $10 \mathrm{~min}$. Authentic standards (Sigma Chemicals) for quantifying amino acids in serum samples were prepared in $0.01-\mathrm{M}$ hydrochloric acid and stored at $-70^{\circ} \mathrm{C}$.

2.3. RT-PCR. Total RNA was extracted from colonic tissues using the TRIZOL reagent kit (Invitrogen, USA). The PrimeScript RT kit with gDNA Eraser (Takara Bio Inc., Qingdao, China) was used to synthesize cDNA, according to the product manual. Primers (Table 1) used in this study were designed under the principles of primer design using Primer 6.0 software (PRIMER-E, New Zealand) and Oligo 5.0 software (Molecular Biology Insights, Inc., USA), based on the gene sequences of the house mouse (Mus musculus) on GenBank. The SYBR Premix Ex Taq kit (Takara, Japan) was used for RT-PCR analysis, and triplicate reactions were performed using the Applied Biosystems 7900HT Fast Real-Time PCR System (Thermo, USA) by the following methods: (1) initial denaturation ( $\left.90^{\circ} \mathrm{C} 30 \mathrm{~s}\right)$; (2) amplification and quantification ( 40 cycles at $95^{\circ} \mathrm{C} 5 \mathrm{~s}, 1 \mathrm{cycle}$ at $60^{\circ} \mathrm{C} 30 \mathrm{~s}$ ). Relative gene expression was calculated with the formula $2^{-(\Delta \Delta \mathrm{Ct})}$.

2.4. Gut Microbiota Analysis. $16 \mathrm{~S}$ rDNA sequencing was used for intestinal microbiota analysis, according to our previous study [20]. Digested matter in the colon cavity was collected, and the DNA was extracted from this material using a Qiagen QIAamp DNA Stool Mini kit. Illumina MiSeq sequencing was used to analyse the $\mathrm{V} 3$ to $\mathrm{V} 4$ regions of bacterial $16 \mathrm{~S}$ rDNA, and the data analysis was performed by Anoru Genomics Technology Co., Ltd., (Beijing, China). Briefly, the library was generated and sequenced to produce 400 base pair/600 base pair single-end reads. Single-end reads were assigned to the samples based on their unique barcode and truncated by the removal of their barcode and the primer sequence. Sequence analysis and operational taxonomic unit (OTU) clustering were performed using UPARSE software (v7.0.1001, https://drive5.com/usearch/) following quality filtering, and sequences with more than $97 \%$ similarity were clustered to the same OTU. RDP Classifier (V2.2, Michigan State University Board of Trustees, East Lansing, MI) was used for species annotations, based on the GreenGene database. MUSCLE software (Version 3.8.31) was used to study the phylogenetic relationships of different OTUs, to examine differences in the dominant species in different samples (groups), and for multiple-sequence alignments. The abundant information in the OTUs was normalized for suvbsequent analysis of the alpha diversity, beta diversity, and the environmental-factor correlation analysis. 


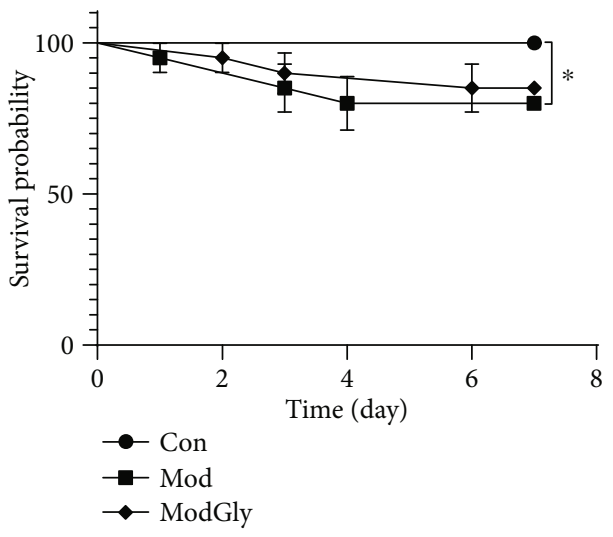

(a)

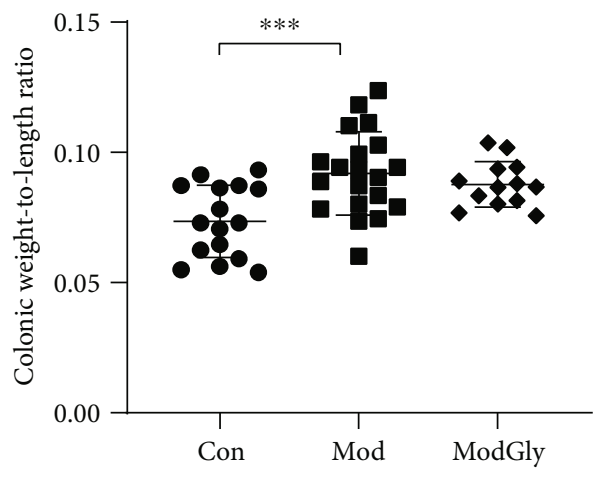

(b)

FIGURE 1: Survival proportions, the ratio of colon weight to length. (a) The survival rate was significantly lower in the Mod group than in the Con group, but was similar in the Mod and ModGly groups. (b) The colonic weight-to-length ratio was greater in the Mod group than in the Con group and was the same in the Mod and ModGly groups. The data are presented as means $\pm \mathrm{SD} ;{ }^{*} P<0.05,{ }^{* *} P<0.01,{ }^{* * *} P<0.001$.

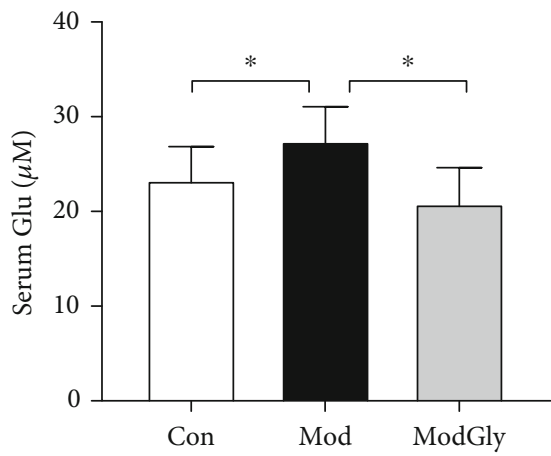

(a)

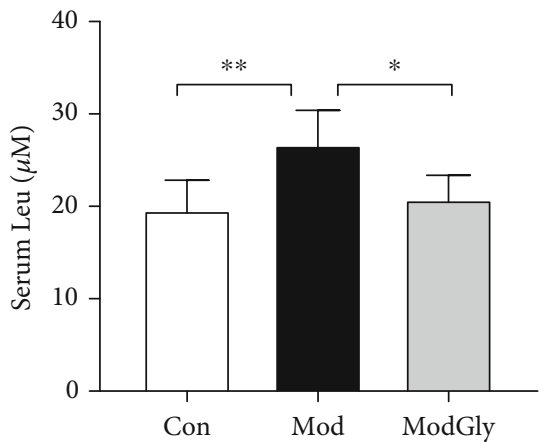

(c)

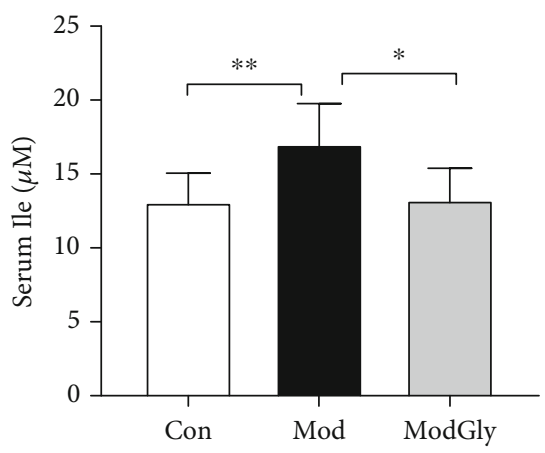

(b)

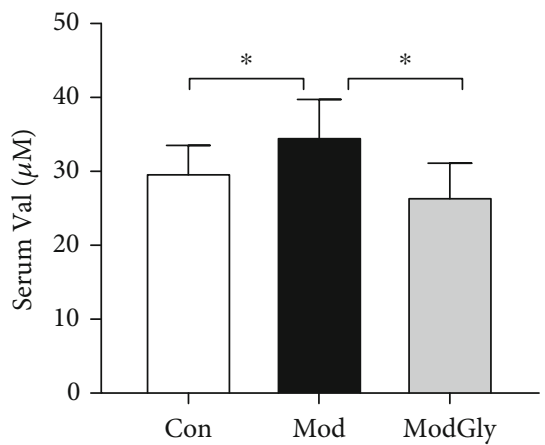

(d)

FIGURE 2: Serum amino acid profiles in the serum. The serum concentrations of Glu, glutamic acid (a), Ile, isoleucine (b), Leu, leucine (c), and Val, valine $(\mathrm{d})$ were significantly higher $(P<0.05)$ in the Mod group than in the Con group and significantly lower $(P<0.05)$ in the Mod group than in the ModGly group. The data are presented as means $\pm \mathrm{SD} ;{ }^{*} P<0.05,{ }^{* *} P<0.01,{ }^{* * *} P<0.001$.

2.5. Statistical Analyses. GraphPad Prism 6.0 (GraphPad Software, Inc., La Jolla, CA) was used for statistical analyses and to create graphs. Statistical analyses between the Con and Mod groups and the Mod and ModGly groups were performed by $t$-test. Results are shown as mean \pm SD. $P<0.05$ was considered significant; ${ }^{*} P<0.05,{ }^{* *} P<$ $0.01, * * * P<0.001$.

\section{Results}

3.1. Survival Proportions and Ratio of Colon Weight to Length. We evaluated the effect of GLY on AA-induced colitis in mice by comparing the survival rate and the ratio of colonic weight to length. The survival rate in the Mod group was significantly lower than that in the CON group 
IL-1 $\beta$

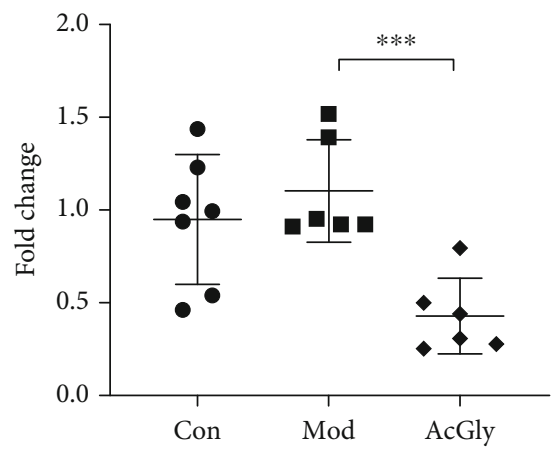

(a)

IFN- $\gamma$

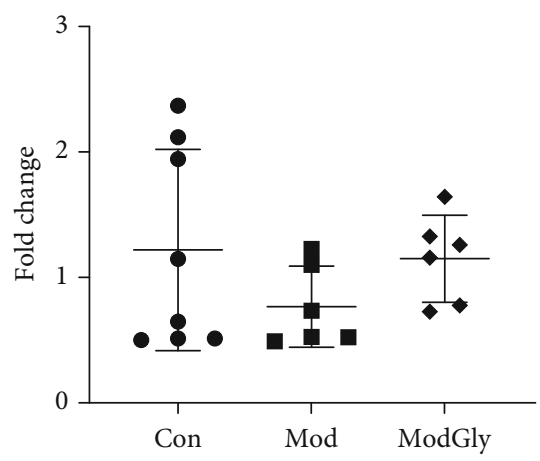

(c)
IL-17

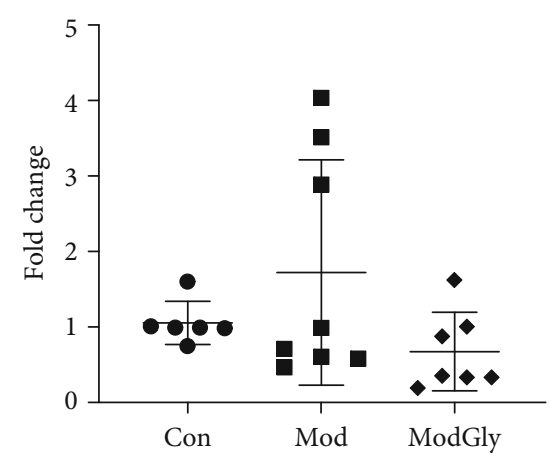

(b)

IL-10

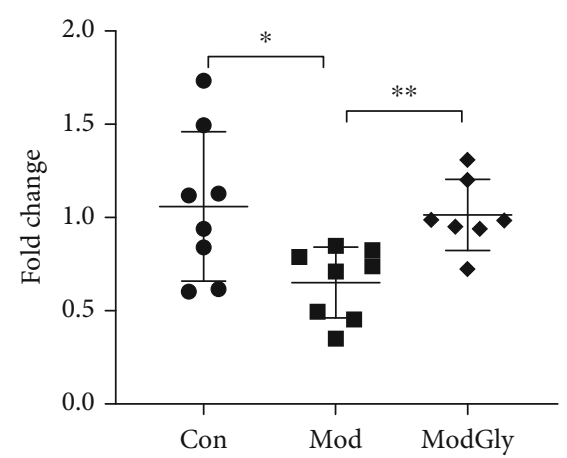

(d)

Figure 3: Colonic expression of IBD-associated cytokines. Real time-polymerase chain reaction analysis was used to measure the colonic expression of IL-1 $\beta$ (a), IL-17 (b), IFN- $\gamma$ (c), and IL-10 (d) genes. AA administration reduced IL-10 expression, whereas Gly inhibited IL$1 \beta$ expression and promoted IL-10 expression. The data are presented as means $\pm \mathrm{SD} ;{ }^{*} P<0.05,{ }^{* *} P<0.01,{ }^{* * *} P<0.001$.

(Figure 1(a)), and the colonic weight/length ratio was increased $(P<0.05)$ (Figure $1(\mathrm{~b}))$. However, Gly did not affect the survival rate or the colonic weight-to-length ratio in the ModGly group (Figures 1(a)-1(b)).

3.2. Serum Amino Acid Profile. The results showed that the serum leucine (Leu), isoleucine (Ile), valine (Val), and glutamic acid (Glu) concentrations were significantly higher $(P<0.05)$ in the Mod group than in the CON group (Figures 2(a)-2(d)), whereas the concentrations of other amino acids were largely unchanged (data not shown). Notably, dietary Gly reduced the serum concentrations of Leu, Ile, Val, and Glu in the Mod group.

3.3. Colonic Expression of IBD-Associated Cytokines. We analyzed the gene expression of colonic proinflammatory cytokines (such as IL-1 $\beta$, IFN- $\gamma$, and IL-17) and inhibitory cytokine (IL-10). Compared with the CON group, the MOD group had significantly reduced expression of IL$10(P<0.05)$ (Figure $3(\mathrm{~d}))$ but had an unaltered expression of proinflammatory cytokines (Figures $3(\mathrm{a})-(\mathrm{c})$ ). In contrast, the ModGly group had significantly increased IL-10 expression (Figure 3(d)) and reduced IL-1 $\beta$ expression (Figure 3(c)).

3.4. Intestinal Microbiota. 16sRNA was analyzed by Illumina high-throughput sequencing to examine the effect of the experimental conditions on the gut microbiota of mice. A total of 260 OTUs were found overall, and 233 of these OTUs were present in all three groups (Figure 4(a)). Twenty-six taxa were detected at the genus level, such as Bacteroides, Prevotella, Helicobacter, Akkermansia, Lactobacillus, and Sutterella (Figure 4(b)). Clostridia, Clostridiales, and Ruminococcaceae were relatively more abundant in both the Con and ModGly groups than in the Mod group (Table 2). The prominent populations in each group were also determined based on linear discriminant analysis coupled with effect-size measurements, which revealed that Clostridia and Clostridiales were dominant in the Con group, Alcaligenaceae, Burkholderiales, and Betaproteobacteria were dominant in the Mod group, and Ruminococcaceae were dominant in the ModGly group (Figure 4(c)). A correlation analysis showed that the serum concentrations of $\mathrm{Val}$ and Leu were negatively correlated with the abundance of Clostridia and Clostridiales (Figure 4(d)). Moreover, colonic IL-10 expression was positively correlated with the abundance of Clostridia, Clostridiales, and IL-1 expression was negatively correlated with the abundance of Ruminococcaceae (Figure 4(e)).

\section{Discussion}

Previous studies have shown that body weight, survival rates, and the ratio of colon weight to colon length decreased in the 

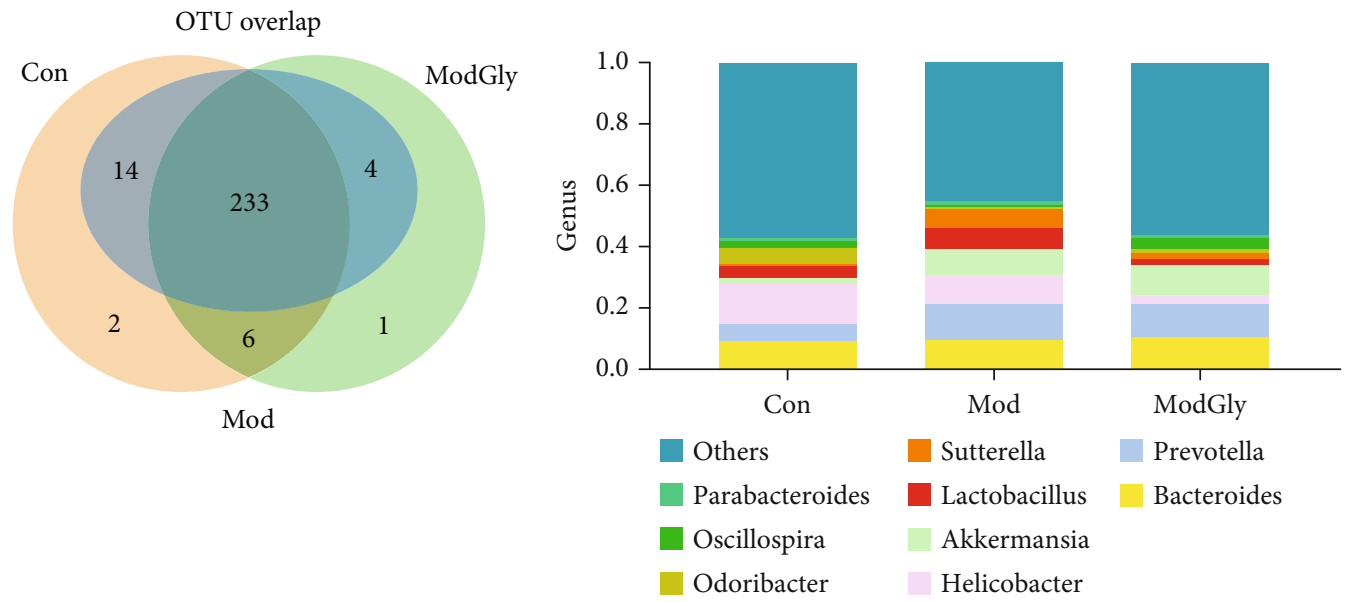

(a)

(b)

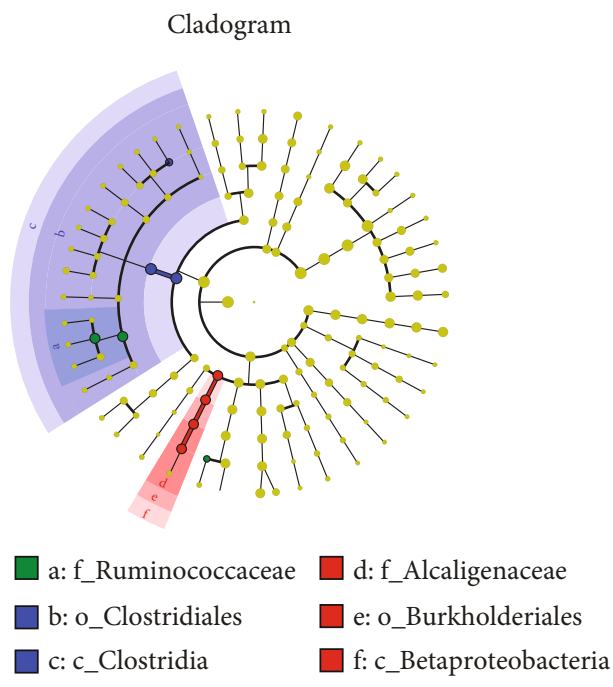

(c)

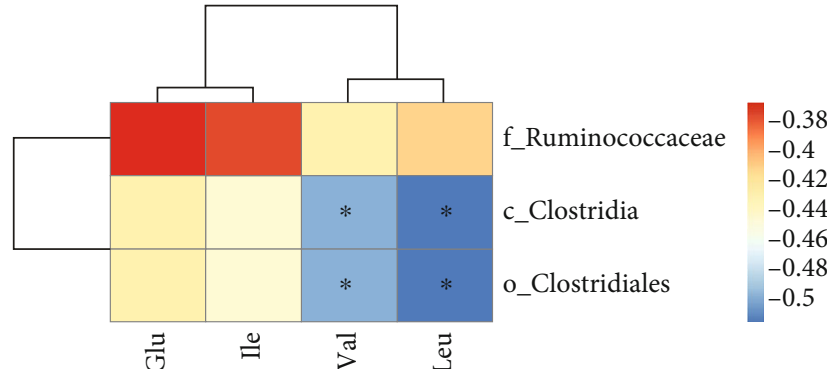

(d)

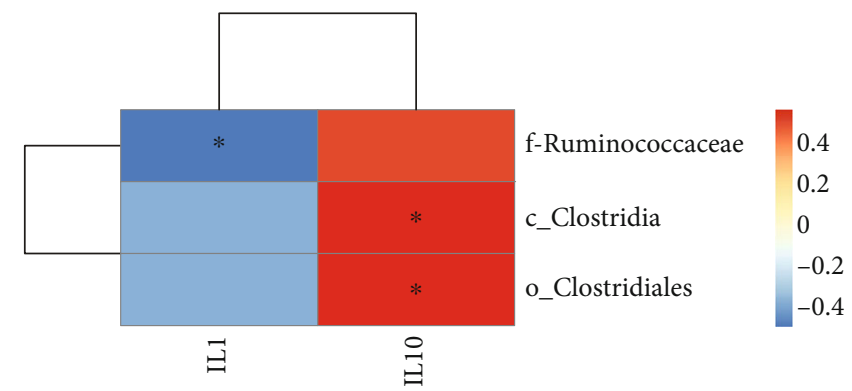

(e)

FIgURE 4: Intestinal microbiota. (a) Two hundred thirty-three OTUs were common to all three groups. (b) Relative contribution of genera in each group. (c) Prominent populations in each: Clostridia and Clostridiales in the Con group, Alcaligenaceae, Burkholderiales, and Betaproteobacteria in the Mod group, and Ruminococcaceae in the ModGly group. (d) The correlation analysis showed that serum concentrations of valine and leucine were negatively correlated with the abundance of Clostridia and Clostridiales (e); IL-10 expression was positively correlated with the abundance of Clostridia, Clostridiales, and IL-1 expression was negatively correlated with the abundance of Ruminococcaceae.

AA-induced IBD mouse model $[17,21]$. It has also been shown that the expression of proinflammatory cytokines (i.e., IL- $1 \beta$, IFN- $\gamma$, and IL-17) is significantly increased in the AA-induced IBD model. Intriguingly, it has been found that dietary supplementation Gly partially restores the above indicators in Wistar rat models of TNBS-or DSS-induced IBD [17]. However, we found that dietary Gly did not have a significant effect on survival rate and colonic weight-to- 
TABLE 2: Taxa with significantly changed relative abundances at the genus level.

\begin{tabular}{lccc}
\hline & Con & Mod & Gly \\
\hline Clostridia & $0.21380^{*}$ & 0.07538 & $0.1699^{\#}$ \\
Ruminococcaceae & $0.03527^{*}$ & 0.01695 & $0.06516^{\#}$ \\
Clostridiales & $0.2138^{*}$ & 0.07538 & $0.2130^{\#}$ \\
\hline
\end{tabular}

The relative abundances of Clostridia, Clostridiales, and Ruminococcaceae were significantly higher in both the Con and ModGly groups than in the Mod group. The data are presented as means $\pm \mathrm{SD} ;{ }^{*} P<0.05$, Con vs. Mod; ${ }^{*} P<0.05$, ModGly vs. Mod.

length ratio in the mouse model with AA-induced IBD. This different result may be attributable to the mouse model and/or the dosage of Gly we used.

Amino acids are necessary for intestinal growth and for the maintenance of mucosal integrity and barrier function [22-24]. Dietary supplementation with amino acids, such as threonine, serine, proline, and cysteine, improves mucin production, protects intestinal epithelial cells, and enhances the health of the intestinal microbiome [25-27]. Some amino acids have been suggested to be suitable as biomarkers for diagnosis and treatment in IBD, such as branched-chain amino acids (BCAAs; i.e., Leu, Ile, and Val) and tryptophan [28]. Concentrations of BCAAs and Glu have also been found to be significantly increased in a model of IBD [7], and these increases could be reversed by dietary Gly. Studies have also reported that BCAAs enhance the intestinal immune system by improving the morphological integrity of the intestinal tract and increasing the production of immunoglobulin. For example, Leu enhances cell proliferation and amino-acid transporter expression by activating the mTOR pathway [29]. However, a high concentration of BCAAs may activate the mTOR and NF- $\kappa$ B pathways and thereby increase oxidative stress and inflammation. Other non-BCAAs have shown beneficial effects: for example, arginine reduces mucosal permeability, inhibits inflammation, and increases iNOS activity in mice with DSS-induced colitis [15]. Moreover, Gly inhibits cytokine secretion in monocytes, macrophages, and neutrophils and regulates $\mathrm{T}$ cell activities [16].

Current evidence suggests that immune disorders contribute to the IBD process via cytokine production in bowel lesions. Many proinflammatory cytokines, such as TNF- $\alpha$ and IL-1/6/22/23, and anti-inflammatory cytokines (such as IL-10) have been studied to determine their roles in IBD [30]. For example, it was found that IL-1 is highly activated in IBD patients and that IL- $1 \beta$ activates CD4+ T cells [31]. It was also found that mice that the lack of IL-10 or IL-10R are sensitive to colitis [32], largely due to the promotion of IL-1 production.

In another study, it was found that dietary Gly supplementation could reverse the increase of colonic IL- $1 \beta$ and the induction of TNF- $\alpha$ gene expression triggered by neutrophil-produced chemokines and inflammatory proteins secreted by macrophages [17]. Similarly, we found in this study that dietary Gly supplementation inhibited IL-1 expression and promoted IL-10 expression (as shown by increased IL-10 mRNA abundance), suggesting the potential of Gly for IBD treatment.

The gut microbiome has been linked to many diseases, such as IBD, obesity, diabetes, and autism [33]. Some studies have reported dramatic changes in the composition of the intestinal microbial community in mice with IBD, which is in keeping with our results. Dietary supplements, such as amino acids, may regulate physiological activity via their effects on gut microbes [34, 35]. In addition, a low-Gly diet is associated with changes in gut flora [36]. In this study, we found that Gly changed the composition of the microbiota, and this has associations with serum concentrations of amino acids and colonic gene expression of IL-1 and IL-10 in IBD models.

Interestingly, Clostridium butyricum, a member of Clostridia and Clostridiales, has been reported to promote the growth of gut IL-10-producing innate immune cells and alleviate colitis in a mouse model by activating Treg and inhibiting activated macrophages [37]. The findings indicate that dietary Gly regulates colonic cytokine expressions, especially that of IL-10, possibly via effects on gut bacteria. However, further studies are needed to investigate this possible mechanism.

In conclusion, our data show that dietary Gly modulated serum amino acid concentrations and gut cytokine expression in AA-induced colitis mice and that this may have occurred via the regulation of the intestinal microbiota. These findings enhance our understanding of the roles of Gly in the metabolism of serum amino acids, intestinal immunity, and its effects on the intestinal microbiota of mice.

\section{Data Availability}

The data used to support the findings of this study are available from the corresponding author upon request.

\section{Conflicts of Interest}

The authors have no conflicts of interest.

\section{Authors' Contributions}

Shuai Chen and Jie Yin designed the experiment, which was performed by Xin Wu and Yongmin Zheng. Jie Ma analyzed the data and prepared tables and figures. Shuai Chen and Xin Wu prepared the manuscript. All of the authors reviewed the manuscript. Xin $\mathrm{Wu}$ and Yongmin Zheng contributed equally to this study.

\section{Acknowledgments}

This study was supported by the National Natural Science Foundation of China (Nos. 31772642, 31672457, and 31672433), the International Partnership Program of Chinese Academy of Sciences (161343KYSB20160008), the Hunan Provincial Science and Technology Department (2017NK2322), the China Postdoctoral Science Foundation (2018M632963), and the Key Programs of Frontier Scientific Research of the Chinese Academy of Sciences (QYZDYSSW-SMC008). 


\section{References}

[1] K. L. Glassner, B. P. Abraham, and E. M. M. Quigley, "The microbiome and inflammatory bowel disease," The Journal of Allergy and Clinical Immunology, vol. 145, no. 1, pp. 16-27, 2020.

[2] G. Doherty, K. H. Katsanos, J. Burisch et al., "European Crohn's and Colitis Organisation topical review on treatment withdrawal ['exit strategies'] in inflammatory bowel disease," Journal of Crohn's and Colitis, vol. 12, no. 1, pp. 17-31, 2018.

[3] M. Schirmer, A. Garner, H. Vlamakis, and R. J. Xavier, "Microbial genes and pathways in inflammatory bowel disease," Nature Reviews Microbiology, vol. 17, no. 8, pp. 497$511,2019$.

[4] G. G. Kaplan, "The global burden of IBD: from 2015 to 2025," Nature Reviews Gastroenterology \& Hepatology, vol. 12, no. 12, pp. 720-727, 2015.

[5] H. Huang, M. Fang, L. Jostins et al., "Fine-mapping inflammatory bowel disease loci to single-variant resolution," Nature, vol. 547, no. 7662, pp. 173-178, 2017.

[6] H. S. P. de Souza, "Etiopathogenesis of inflammatory bowel disease," Current Opinion in Gastroenterology, vol. 33, no. 4, pp. 222-229, 2017.

[7] Y. Liu, X. Wang, and C. A. Hu, "Therapeutic potential of amino acids in inflammatory bowel disease," Nutrients, vol. 9, no. 9, p. 920, 2017.

[8] N. Taghipour, N. Mosaffa, H. A. Aghdaei et al., "Immunomodulatory effect of Syphacia obvelata_in treatment of experimental DSS-induced colitis in mouse model," Scientific Reports, vol. 9, no. 1, article 19127, 2019.

[9] D. Low, D. D. Nguyen, and E. Mizoguchi, "Animal models of ulcerative colitis and their application in drug research," Drug Design, Development and Therapy, vol. 7, pp. 13411357, 2013.

[10] X. Roblin, N. Williet, G. Boschetti et al., "Addition of azathioprine to the switch of anti-TNF in patients with IBD in clinical relapse with undetectable anti-TNF trough levels and antidrug antibodies: a prospective randomised trial," Gut, vol. 69, no. 7, pp. 1206-1212, 2020.

[11] T. Gjuladin-Hellon, M. Gordon, Z. Iheozor-Ejiofor, and A. K. Akobeng, "Oral 5-aminosalicylic acid for maintenance of surgically-induced remission in Crohn's disease," Cochrane Database of Systematic Reviews, vol. 6, article CD008414, 2019.

[12] H. Courthion, T. Mugnier, C. Rousseaux, M. Möller, R. Gurny, and D. Gabriel, "Self-assembling polymeric nanocarriers to target inflammatory lesions in ulcerative colitis," Journal of Controlled Release, vol. 275, pp. 32-39, 2018.

[13] V. Dubinsky, L. Reshef, N. Bar et al., "Predominantly antibiotic-resistant intestinal microbiome persists in patients with pouchitis who respond to antibiotic therapy," Gastroenterology, vol. 158, no. 3, pp. 610-624.e13, 2020.

[14] K. Wang, X. Jin, Q. Li et al., "Propolis from different geographic origins decreases intestinal inflammation and Bacteroides spp. populations in a model of DSS-induced colitis," Molecular Nutrition \& Food Research, vol. 62, no. 17, 2018.

[15] L. A. Coburn, X. Gong, K. Singh et al., "L-arginine supplementation improves responses to injury and inflammation in dextran sulfate sodium colitis," PLoS One, vol. 7, no. 3, article e33546, 2012.
[16] J. Van Den Eynden, S. SahebAli, N. Horwood et al., "Glycine and glycine receptor signalling in non-neuronal cells," Frontiers in Molecular Neuroscience, vol. 2, no. 9, 2009.

[17] I. Tsune, K. Ikejima, M. Hirose et al., "Dietary glycine prevents chemical-induced experimental colitis in the rat," Gastroenterology, vol. 125, no. 3, pp. 775-785, 2003.

[18] K. Sugihara, T. L. Morhardt, and N. Kamada, "The role of dietary nutrients in inflammatory bowel disease," Frontiers in Immunology, vol. 9, article 3183, 2019.

[19] S. Chen, M. Wang, L. Yin et al., "Effects of dietary tryptophan supplementation in the acetic acid-induced colitis mouse model," Food \& Function, vol. 9, no. 8, pp. 41434152, 2018.

[20] S. Chen, B. Tan, Y. Xia et al., "Effects of dietary gammaaminobutyric acid supplementation on the intestinal functions in weaning piglets," Food \& Function, vol. 10, no. 1, pp. 366378, 2019.

[21] D. K. Podolsky, "Inflammatory bowel disease," New England Journal of Medicine, vol. 347, no. 6, pp. 417-429, 2002.

[22] F. He, C. Wu, P. Li et al., "Functions and signaling pathways of amino acids in intestinal inflammation," BioMed Research International, vol. 2018, Article ID 9171905, 13 pages, 2018.

[23] W. Tang, J. Wu, S. Jin et al., "Glutamate and aspartate alleviate testicular/epididymal oxidative stress by supporting antioxidant enzymes and immune defense systems in boars," Science China. Life Sciences, vol. 63, no. 1, pp. 116-124, 2020.

[24] Q. Guo, F. Li, Y. Duan et al., "Oxidative stress, nutritional antioxidants and beyond," Science China Life Sciences, vol. 63, no. 6, pp. 866-874, 2020.

[25] M. Faure, C. Mettraux, D. Moennoz et al., "Specific amino acids increase mucin synthesis and microbiota in dextran sulfate sodium-treated rats," The Journal of Nutrition, vol. 136, no. 6, pp. 1558-1564, 2006.

[26] G. Guan, S. Ding, Y. Yin, V. Duraipandiyan, N. A. Al-Dhabi, and G. Liu, "Macleaya cordata extract alleviated oxidative stress and altered innate immune response in mice challenged with enterotoxigenic Escherichia coli," Science China Life Sciences, vol. 62, no. 8, pp. 1019-1027, 2019.

[27] M. Bai, H. Liu, K. Xu et al., "Compensation effects of coated cysteamine on meat quality, amino acid composition, fatty acid composition, mineral content in dorsal muscle and serum biochemical indices in finishing pigs offered reduced trace minerals diet," Science China Life Sciences, vol. 62, no. 11, pp. 1550-1553, 2019.

[28] Y. Tamura, H. Ohta, Y. Kagawa et al., "Plasma amino acid profiles in dogs with inflammatory bowel disease," Journal of Veterinary Internal Medicine, vol. 33, no. 4, pp. 16021607, 2019.

[29] M. Coëffier, S. Claeyssens, M. Bensifi et al., "Influence of leucine on protein metabolism, phosphokinase expression, and cell proliferation in human duodenum," The American Journal of Clinical Nutrition, vol. 93, no. 6, pp. 1255-1262, 2011.

[30] I. Marafini, S. Sedda, V. Dinallo, and G. Monteleone, "Inflammatory cytokines: from discoveries to therapies in IBD," Expert Opinion on Biological Therapy, vol. 19, no. 11, pp. 1207-1217, 2019.

[31] D. S. Shouval, A. Biswas, Y. H. Kang et al., "Interleukin $1 \beta$ Mediates Intestinal Inflammation in Mice and Patients With Interleukin 10 Receptor Deficiency," Gastroenterology, vol. 151, no. 6, pp. 1100-1104, 2016. 
[32] J. M. Lanis, E. E. Alexeev, V. F. Curtis et al., "Tryptophan metabolite activation of the aryl hydrocarbon receptor regulates IL-10 receptor expression on intestinal epithelia," Mucosal Immunology, vol. 10, no. 5, pp. 1133-1144, 2017.

[33] R. B. Sartor and G. D. Wu, "Roles for intestinal bacteria, viruses, and fungi in pathogenesis of inflammatory bowel diseases and therapeutic approaches," Gastroenterology, vol. 152, no. 2, pp. 327-339.e4, 2017.

[34] S. Chen, P. Bin, W. Ren et al., "Alpha-ketoglutarate (AKG) lowers body weight and affects intestinal innate immunity through influencing intestinal microbiota," Oncotarget, vol. 8, no. 24, pp. 38184-38192, 2017.

[35] S. Chen, W. K. Ren, P. Bin et al., "Alpha-ketoglutarate lowers body weight and affects intestinal immunity through intestinal microbiota," Amino Acids, vol. 47, no. 8, pp. 1642-1642, 2015.

[36] V. M. Koistinen, O. Kärkkäinen, K. Borewicz et al., "Contribution of gut microbiota to metabolism of dietary glycine betaine in mice and in vitro colonic fermentation," Microbiome, vol. 7, no. 1, p. 103, 2019.

[37] A. Hayashi, T. Sato, N. Kamada et al., "A single strain of Clostridium butyricum induces intestinal IL-10-producing macrophages to suppress acute experimental colitis in mice," Cell Host \& Microbe, vol. 13, no. 6, pp. 711-722, 2013. 